\title{
Use and management of traditional medicinal plants by Maale and Ari ethnic communities in southern Ethiopia
}

Berhane Kidane ${ }^{1,2^{*}}$, Tinde van Andel ${ }^{3}$, Laurentius Josephus Gerardus van der Maesen ${ }^{3}$ and Zemede Asfaw ${ }^{4}$

\begin{abstract}
Background: Around $80 \%$ of the people of Ethiopia are estimated to be relying on medicinal plants for the treatment of different types of human health problems. The purpose of this study was to describe and analyse the use and management of medicinal plants used for the treatment of human health problems by the Maale and Ari communities in southern Ethiopia.
\end{abstract}

Methods: Quantitative and qualitative ethnobotanical field inquiries and analytical methods including individual and focus group discussions (18), observations, individual interviews $(n=74)$, preference ranking and paired comparison were used. Data were collected in three study sites and from two markets; the latter surveyed every 15 days from February 2011 to February 2012.

Results: A total of 128 medicinal plant species, belonging to 111 genera and 49 families, used as herbal medicine by Maale and Ari communities were documented. Predominantly harvested plant parts were leaves, which are known to have relatively low impact on medicinal plant resources. Species with high familiarity indices included Solanum dasyphyllum, Indigofera spicata, Ruta chalepensis, Plumbago zeylanica and Meyna tetraphylla. Low Jaccards similarity indices $(\leq 0.33)$ indicated little correspondence in medicinal plant use among sites and between ethnic communities. The dominant ways of medicinal plant knowledge acquisition and transfer is vertical: from parents to children through oral means. Gender and site significantly influenced the number of human medicinal plants known currently in the study sites. Age was only a factor of significance in Maale. Marketing of medicinal plants harvested from wild and semi-wild stands is not common. Expansion of agricultural land and lack of cultivation efforts by local communities are mentioned by locals to affect the availability of medicinal plant resources.

Conclusion: S. dasyphyllum, I. spicata, P. zeylanica, M. tetraphylla, and Oxalis radicosa need to be considered for phytochemical and pharmacological testing to verify their efficacy and determine their dosages. Land use planning and development initiatives in the area and beyond need to sharply focus on strategies that could alleviate the major threats affecting medicinal plant resources in the landscape and encourage their cultivation to enhance their availability and complement ex-and in-situ conservation.

\section{Background}

Ethiopia is a country with regional differences in access to health services [1]. It is estimated that traditional medicine (about $95 \%$ herbal) is used by $80 \%$ of the Ethiopian population for the treatment of different types of human health problems [2]. Medicinal plants are used as a major source

\footnotetext{
* Correspondence: Berhanekid19@yahoo.com

'Ethiopian Institute of Agricultural Research, Forestry Research Center, P.O. Box 58532, Addis Ababa, Ethiopia

${ }^{2}$ Biosystematics Group, Wageningen University and Research Center, P.O. Box 647, 6700 Wageningen, AP, The Netherlands

Full list of author information is available at the end of the article
}

for health promotion, prevention and cure [3,4]. The traditional use of medicinal plants by most Ethiopians in health care system is generally ascribed to the incomplete coverage of the modern medical system, unaffordable and not always available prescription drugs [5-7] and the widespread belief in the effectiveness of herbal medicine $[8,9]$.

Medicinal plant knowledge is shaped by the ecological diversity of the country [10], known to be site-specific [11] and varies across peoples with different religious, linguistic and cultural backgrounds [7]. In Ethiopia, there 
are over 70 ethnic communities, residing in different ecological regions [12] and the studies so far have shown extensive medicinal plant knowledge, acquired through centuries of experience. Although several studies have been conducted on medicinal plants throughout the country e.g. [13-18], the full wealth of this knowledge has not yet been sufficiently studied. We therefore document medicinal plants used by the Maale and Ari communities less studied Ethiopian communities and evaluate similarities and differences among sites and between the two communities.

Ethiopian farmers' knowledge on medicinal plants may be influenced by certain demographic characteristics. Awas [11] and Giday et al. [16] showed that gender and age significantly affected farmers' knowledge on traditional medicine. However, in other farming communities, gender and age had no significant effect on useful plant knowledge [19]. In order to effectively preserve indigenous knowledge, we need to find out whether socio-demographic factors (age, gender, religion, educational level, family size) or locality affect the level of medicinal plant knowledge among the Maale and Ari communities.

Traditionally used medicinal plants and associated knowledge are disappearing at an alarming rate [20]. Natural and anthropogenic factors contribute to these losses but threatening factors may vary from one region to the other [17]. Therefore, we want to understand factors that threaten local traditional medicinal plant resources and knowledge, which are important for decision makers for their policy formulation and analysis. Mechanisms of medicinal plant knowledge acquisition and transfer affect knowledge continuity within a community. Most studies conducted in Ethiopia so far have shown that the major mechanism for transfer of ethnomedicinal knowledge is oral [20], although Fassil [8] indicated existence of pharmacopias (ancient written medicinal plant knowledge) in monasteries in the northern highlands of Ethiopia. In order to preserve herbal medicine traditions, we need to reveal the local mechanisms of knowledge transfer.

Marketing of medicinal plants may have implications on natural resources, depending on the species marketed, the type of plant part harvested, volumes sold and cultivation efforts of commercial species. A few of the ethnobotanical studies in Ethiopia attempted to highlight the issue of marketing of medicinal plants $[21,16]$. However, the studies were of cross-sectional nature and did not conduct systematic repeated visits and data collection and elements that affect the marketing of traditional medicinal plants were hardly addressed. In this paper, we describe and analyse the use and management of medicinal plants by Maale and Ari communities by answering the following research questions:
1) Which plant species are used as medicines at different sites by the two communities and for what purposes?

2) What are the mechanisms of herbal medicinal plant knowledge transfer among different social groups?

3) Which demographic factors significantly influence medicinal plant knowledge?

4) How do the two communities differ in their use and handling of medicinal plants?

5) What factors threaten medicinal plant resources in the study area?

6) Which medicinal plants are commercialized and what elements affect their marketing?

\section{Materials and methods}

\section{Study area and selection of informants}

The study was conducted in the Maale and Debub Ari districts of southern Ethiopia, where the Maale and Ari communities reside. The study area is located at about $750 \mathrm{~km}$ south of the Ethiopian capital, Addis Ababa.

A reconnaissance survey was conducted from July to August 2010 in the two study districts. Well settled areas by the two ethnic communities within the two districts studied. Detailed fieldwork was conducted from August 2010 to October 2012. Prior to detailed data collection, individual and focus group discussions (18) with informants were conducted. At each kebele (the lowest formal administrative unit in rural Ethiopia), 10 to 12 informants from different socio-demographic groups were involved in the focus group discussions. In general we used stratified simple random sampling for the selection of the study sites, study kebeles and study participants. The stratifying variables were ethnic communities and altitude. The three study sites selected for detailed formal survey were Maale, Ari 1 and Ari 2 (Figure 1). For each site, two kebeles (Shole teka and Beneta in Maale, Kure and Geza in Ari 1 and Pilla and Metser in Ari 2) were randomly selected (Figure 1). The Ari 1 and Maale study kebeles were selected from the area with altitude of 500-1500 m.a.s.l. However, the Ari 2 study site is located within altitude ranging from 1500-2500 with mean altitude of $1700 \mathrm{~m}$. Despite the presence of areas with altitude ranging from 1500-2500 in Maale district, the area was not included in the study because there are no substantial settlements.

Acacia - Commiphora and Combretum - Terminalia woodland is characterizes the remnant vegetation of Maale study site; the mean altitude of the selected study site is 1350 m.a.s.l. The remnant vegetation of Ari 1 is characterized by moist evergreen Afromontane forest and Combretum - Terminalia woodland [22] with a mean altitude of 1330 m.a.s.l. The forest area is converted to agricultural land, with scattered trees in the fields and home gardens of Ari 2. The language of Ari is Araf, while the Maale language is spoken by the Maale communities. 


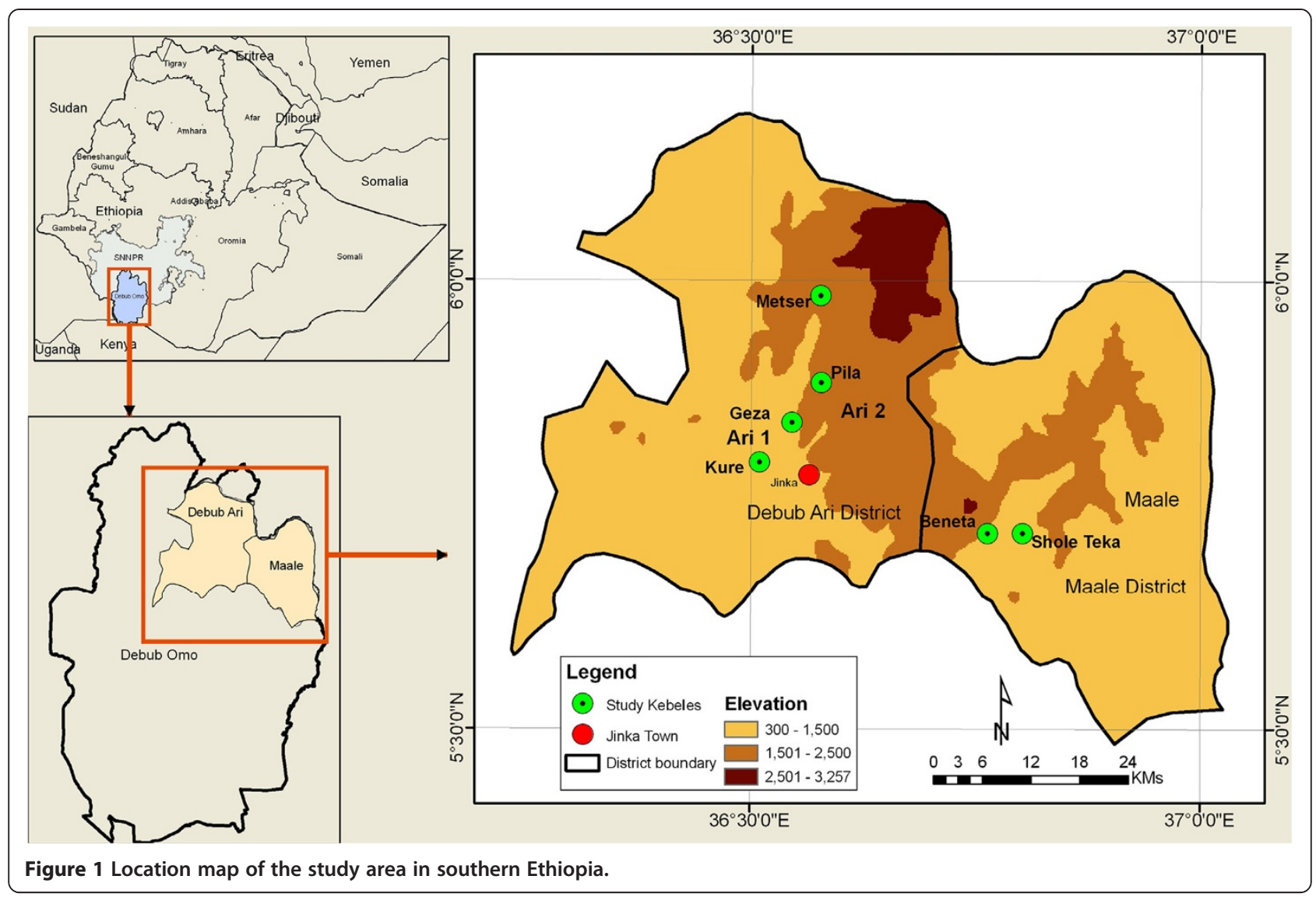

The 2007 population census shows that there were 84657 in Maale and 212389 in Debub Ari [23].

A total of 74 study participants (24 from Ari 1, 24 from Ari 2 and 26 from Maale), belonging to the two ethnic communities were considered for the interviews. The age range of randomly selected study participants lied between 20 and 60 except a single participant who was older: 70 years. Oral informed consent was obtained from each participant prior to conducting the interviews.

\section{Ethical approval}

Written permission (approval) was obtained from South Omo "zonal" council and also from the Maale and Debub Ari district council offices before the start of the study.

\section{Consent}

Oral and also signed informed consent were obtained from the study participants for the publication of this report and any accompanying images.

\section{Data collection}

We used semi-structured interviews, field observations, preference ranking and paired comparison following the standard ethnobotanical methods and procedures as given by various authors [24-26] to collect information on various aspects of medicinal plants in the two districts. The semi-structured interviews contained questions on common health issues, medicinal plants, plant parts used, preparation and application methods, dosage, and possible threats to medicinal plant resources. Moreover, study participants were asked how they acquired medicinal plant knowledge and whether they were willing to transfer this knowledge. Each informant was visited at least two times during the study period in order to validate the information provided. Following the recommended procedures by Alexiades [25], responses of the study participants that contradicted with each other were not considered for analysis.

Voucher specimens of medicinal plants were collected with the assistance of key informants following standard botanical procedures. Preliminary plant identification was done in the field while collecting and further identification and confirmation was done at the National Herbarium (ETH) of Addis Ababa University (AAU) using taxonomic keys provided in the relevant volumes of the Flora of Ethiopia and Eritrea [27-33]. Finally, the identified specimens were deposited at the ETH. Scientific names were checked for accuracy by means of the Plant List [34].

Between February 2011 and February 2012, surveys were carried out at Beneta market (Maale area) and 
Jinka market (Debub Ari). Markets were visited every fifteen days and all medicinal plants sold were documented with their price, source and additional trade information.

\section{Data analysis}

Descriptive statistics were used to calculate average numbers of medicinal plants and illness types listed by the study participants, and to quantify acquisition and transfer of traditional knowledge. In Maale, ten key informants were selected to participate in a preference ranking exercise following Martin [24] for eight plants used to treat the most frequently cited health problem (in this case ascariasis). Key informants ranked plant species according to their perceived efficacy: the most effective being given a value of one, and the least effective a value of eight. Priority ranking was used to classify major issues that affected the availability of medicinal plants in the study sites.

The calculated Jaccard's similarity indices [35] were used to compare similarity of medicinal plant knowledge among the studied communities. This index uses plant presence/positive reply or absence/negative reply data sets and is expressed as:

$$
J=\frac{c}{a+b+c}
$$

Where JI is the Jaccard similarity index, $\mathrm{c}$ is the number of species shared by the study sites, a is the number of species in study site A only and b is the number of species in study site B only. The JI values range between 0 and 1, whereby a value of 1 indicates complete similarity.

Familiarity index (FI) was used as an indicator of the popularity of a species [36]. FI was defined as the number of respondents that mentioned a species for a specific use, divided by the total number of respondents. The value of FI varies between 0 and 1, whereby a value of 1 represents the highest familiarity of a medicinal plant in the study site.

$$
F I=\frac{\text { Frequency of a given species mentioned as a medicine }}{\text { Total number of respondents }}
$$

Multiple regression analysis was employed to reveal demographic factors that predicted traditional knowledge [37]. We used the number of plants known as dependent variable and considered age, gender, religion, education level, family size and geographic location as explanatory variables. Variables that were highly correlated $(r \geq 0.9)$ were not included in the model [37]. Independent t-tests were employed to compare the differences between gender and age groups. All statistical methods were carried out in the program SPSS 20.0.

\section{Results and discussion}

\section{Medicinal plants reported}

A total of 128 medicinal plant species, belonging to 111 genera and 49 families were reported by Maale and Ari communities for the treatment of 48 different types of health problems (see Table 1). The family Lamiaceae was represented by the highest number of species (19) followed by Leguminosae (10 species), Acanthaceae, Solanaceae, Cucurbitaceae, and Malvaceae (each $8 \mathrm{spp}$ ) and Rubiaceae and Compositae (6 spp each). The highest number (92 spp) was reported in Maale, while 54 species were jointly documented in Ari 1 and Ari 2, of which 18 species were shared. Medicinal plants were used as the first line of treatment by $96 \%, 83 \%$, and $88 \%$ of the respondents of Maale1, Ari 1 and Ari 2 respectively. This indicates that traditional medicine plays a significant role in the primary health care system of the Maale and Ari communities.

Malaria, diarrhoea, ascariasis and amoebiasis were the most frequently cited health problems in Maale. Ascariasis is a helminthic human infection caused by Ascaris lumbricoides, a large roundworm. It is found worldwide with highest prevalence in tropical and subtropical regions, and in areas with inadequate sanitation [38,39]. Malaria, headache, stomach ache and diarrhoea were the main cited ailments in Ari 1 and Ari 2. Respondents said they identified and diagnosed the type of ailments by visual observation of the human body. Yellow, white of the eyes, for example, indicated liver health problem. Lulekal et al. [40] reported similar ways of diagnosis among traditional healers in Mana Angetu, southeastern Ethiopia. On the other hand, tape worm and ascariasis were diagnosed by observation of the worms in human faeces by the patients themselves or elders in the case of young children.

The average number of medicinal plants cited by each study participant of different age and gender groups is displayed in Table 2. The highest numbers of species were mentioned by participants from the Maale ethnic community. Moreover, in all study sites the results revealed that male participants mentioned a higher number of medicinal plants than female ones (t-test, $\mathrm{p}<0.05$ ). Our results are in agreement with the study results reported for the Bench ethnic communities in south-western Ethiopia by Giday et al. [16] that they found that the male study participants to have greater plant knowledge than females, because boys were favoured for the transfer of medicinal plant knowledge.

In all study sites more medicinal plants were reported by participants over 40 years of age than by younger ones but this difference was significant for the Maale site. Awas [11] found that older people knew more than the youngsters in his study of the Kefficho people, in south-western Ethiopia. This may be due to the fact that knowledge tends to be accumulated through time. The 
Table 1 Medicinal plants used in Maale and Ari communities with plant parts used, growth form and applications

\begin{tabular}{|c|c|c|c|c|c|c|c|c|c|}
\hline $\mathrm{N}_{-}^{\circ}$ & $\begin{array}{l}\text { Vernacular names: } \\
\text { Maale (M), Araf (A) }\end{array}$ & Ailments & Scientific name & Family Name & Voucher № & Parts used & $\begin{array}{l}\text { Growth } \\
\text { form }\end{array}$ & $\begin{array}{l}\text { Application } \\
\text { route }\end{array}$ & $\begin{array}{l}\text { Study } \\
\text { sites }\end{array}$ \\
\hline 1 & Tsinkaso (M) & Eye disease (infection), Headache & Barleria ventricosa Hochst. ex Nees & Acanthaceae & 210,356 & Leaves & Herb & Topical & M \\
\hline 2 & Moro Golodo (M) & Gastritis & Blepharis maderaspatensis (L.) Roth. & Acanthaceae & 234,637 & Whole & Herb & Oral & M \\
\hline 3 & Golodo (M) & Oral trash, Gastritis, Malnutrition & Justicia bizuneshiae Ensermu & Acanthaceae & 357 & Leaves & Herb & Oral & M \\
\hline 4 & Busino (M) & Amoebiasis, Stomach ache, Diarrhea & Hypoestes forsskaolii (Vahl) R. Br. & Acanthaceae & 30 & Root & Herb & Oral & M \\
\hline 5 & Kati Murso (M) & Constipation, Ascariasis & Thunbergia alata Boj. ex Sims & Acanthaceae & 232,366, 391 & whole & Climber & Oral & M \\
\hline 6 & Majimaylo(M) & Tape worm & Celosia trigyna $\mathrm{L}$. & Amaranthaceae & 679 & Leaves & Herb & Oral & M \\
\hline 7 & Tsami shinkurt (A) & Stomach ache & Allium sativum $\mathrm{L}$. & Amaryllidaceae & - & Bulb & Herb & Oral & $\begin{array}{l}\mathrm{A} 1 \\
\mathrm{~A} 2\end{array}$ \\
\hline 8 & Salvano (M) & Ascariasis & Ozoroa insignis Delile & Anacardiaceae & $\begin{array}{l}177224289 \\
302\end{array}$ & Stem bark & Tree & Oral & M \\
\hline 9 & Kubri (M) & $\begin{array}{l}\text { Diarrhea, Toothache, Food } \\
\text { poisoning, Vomiting }\end{array}$ & Rhus natalensis Krauss & Anacardiaceae & 28 & Leaves & Shrub & Oral & M \\
\hline 10 & Muralatse (M) & Food poisoning, vomiting & Uvaria leptocladon Oliv. & Annonaceae & 24 & Leaves & Shrub & Oral & M \\
\hline 11 & Afi Deshe (A) & Evil eye & Agrocharis melanantha Hochst. & Apiaceae & 309 & Leaves & Herb & & A 2 \\
\hline \multirow[t]{2}{*}{12} & $\begin{array}{l}\text { Etsewayo (M), } \\
\text { Ountinkam (A) }\end{array}$ & Gastritis, Headache Evil eye & Centella asiatica (L.) Urban & Apiaceae & 36,128 & whole & Herb & Oral & $\begin{array}{l}M, A 1 \\
A 2\end{array}$ \\
\hline & & Swelling & Centella asiatica (L.) Urban & & & Whole & Herb & Topical & \\
\hline \multirow[t]{4}{*}{13} & Ambelto (M), Almi (A) & Tonsilites & Carissa spinarum L. & Apocynaceae & 33,119 & Leaves & Tree & Oral & $M, A 1$ \\
\hline & & Snake protection & Carissa spinarum L. & & & Root & Tree & Smoke & \\
\hline & & Stomach ache, & Carissa spinarum L. & & & Root & Tree & Oral & \\
\hline & & Evil eye & Carissa spinarum L. & & & Root & Tree & Inhale & \\
\hline \multirow[t]{2}{*}{14} & Pijie (M) & Ascariasis & Pergularia daemia (Forssk.) Chiov. & Apocynaceae & 304 & Root & Climber & Oral & M \\
\hline & & Malnutrition (Child) & Pergularia daemia (Forssk.) Chiov. & & & Leaves & & Oral & M \\
\hline \multirow[t]{2}{*}{15} & Metse (M) & Retained placenta & Leptadenia hastata (Pel's.) Decne. & Apocynaceae & 101 & Leaves & Shrub & Oral & M \\
\hline & & Liver disease(Ara) & Leptadenia hastata (Pel's.) Decne. & & & Leaves & & Inhale & \\
\hline 16 & Zolpe (M) & Liver disease (Ara) & Stereospermum kunthianum Cham. & Bignoniaceae & 282,402 & Leaves & Tree & Inhale & M \\
\hline 17 & Achenti (A) & Stomach ache & Cynoglossum lanceolatum Forssk. & Boraginaceae & 2 & Root & Herb & Oral & A2 \\
\hline 18 & Kolpo (M) & Common cold & Brassica carinata A. Braun & Brassicaceae & & Leaves & Herb & Oral & M \\
\hline 19 & Feto & Common cold & Lepidium sativum L. & Brassicaceae & - & Seed & Herb & Oral & A2 \\
\hline \multirow[t]{2}{*}{20} & Afi Deshe (A) & Evil eye & Monopsis stellarioides (Presl) Urb. & Campanulaceae & 246 & Leaves & Herb & Oral & A2 \\
\hline & Guni deshe (A) & Snake bite & Monopsis stellarioides (Presl) Urb. & & & & & & \\
\hline 21 & Chedi (A) & Oral trash & Garcinia livingstonei T.Anders. & Clusiaceae & 14 & Fruit & Tree & Topical & A2 \\
\hline 22 & Yemdir Berbere (A) & Tooth ache, Tonsillitis & Acmella caulirhiza Delile & Compositae & 89,313 & Flower & Herb & Topical & A1 \\
\hline
\end{tabular}


Table 1 Medicinal plants used in Maale and Ari communities with plant parts used, growth form and applications (Continued)

\begin{tabular}{|c|c|c|c|c|c|c|c|c|c|}
\hline \multirow[t]{2}{*}{23} & \multirow[t]{2}{*}{ Dunko (M), Duno (A) } & Stomach ache & Artemisia absinthium L. & Compositae & - & $\begin{array}{l}\text { Leaves with } \\
\text { stem }\end{array}$ & Herb & Oral & \multirow[t]{2}{*}{$M, A 1$} \\
\hline & & Evil eye, Sudden disease, Headache & Artemisia absinthium L. & & & $\begin{array}{l}\text { Leaves with } \\
\text { stem }\end{array}$ & & Oral/Inhale & \\
\hline 24 & Hachenti (M) & Diarrhea & Bidens pilosa $\mathrm{L}$. & Compositae & 233 & Root & Herb & Oral & M \\
\hline 25 & Azi deshe $(A)$ & Swelling(eti) & Conyza gouanii (L.) Willd. & Compositae & 311 & Leaves & Herb & Topical & $A 2$ \\
\hline \multirow[t]{2}{*}{26} & Rebasho (M) & Rheumatism & Tagetes minuta L. & Compositae & 203 & Leaves & Herb & Topical & M \\
\hline & & Amoebiasis & Tagetes minuta L. & & & Leaves & Herb & Oral & \\
\hline 27 & Gera (A) & Malaria & Vernonia amygdalina Delile & Compositae & - & leaves & shrub & Oral & $\mathrm{M}, \mathrm{A} 1$ \\
\hline 28 & Kwakuch deshe (A) & Skin disease ('Kwakucha) & Cuscuta campestris Yuncker & Convolvulaceae & 259 & $\begin{array}{l}\text { Leaves with } \\
\text { succulent stem }\end{array}$ & Herb & Topical & A1 \\
\hline 29 & Lago (M) & $\begin{array}{l}\text { Malnutrition (Child) Diarrhea, } \\
\text { Hheart disease, Gastritis }\end{array}$ & Ipomoea obscura (L.) Ker-Gawl. & Convolvulaceae & 211 & Leaves & $\begin{array}{l}\text { Herb/ } \\
\text { climber } \\
\text { type }\end{array}$ & Oral & M \\
\hline 30 & Welanke (M) & Liver disease & Ipomoea spathulata Hall.f. & Convolvulaceae & 173,404 & Leaves & Herb & Inhale & M \\
\hline 31 & Kamakarsho (M) & Ascariasis & Combretum aculeatum Vent. & Combretaceae & 287,504 & Leaves & Shrub & Oral & M \\
\hline \multirow[t]{2}{*}{32} & Gaal (M) & Typoid & Terminalia brownii L. & Combretaceae & & Leaves & Tree & Oral & M \\
\hline & & Snake bite & Terminalia brownii L. & & & Bark & & Oral/Topical & \\
\hline \multirow[t]{2}{*}{33} & Hachirindo (M) & Lower extremity weakness & Cucumis dipsaceus Ehrenb. ex Spach & Cucurbitaceae & 15 & Leaves & Herb & Topical & M \\
\hline & & Amoebiasis & Cucumis dipsaceus Ehrenb. ex Spach & & & Whole & & Oral & \\
\hline \multirow[t]{2}{*}{34} & $\begin{array}{l}\text { Botayilashe (M), } \\
\text { Bota (A) }\end{array}$ & Tape worm & Cucurbita pepo L. & Cucurbitaceae & - & Seed & Herb & Oral & $M, A 1$ \\
\hline & Bota & Tape worm & Cucurbita pepo L. & & & Seed & Herb & Oral & \\
\hline 35 & Shuntee (M) & Mouth wound & Kedrostis foetidissima (Jacq.) Cogn & Cucurbitaceae & 84 & Leaves & Herb & Topical & M \\
\hline 36 & Choko (M) & Amoebiasis & Momordica foetida Schumach. & Cucurbitaceae & 204,242 & Whole & Herb & Oral & M \\
\hline \multirow[t]{2}{*}{37} & Najie (M) & Evil eye & Momordica pterocarpa Hochst. ex A. Rich. & Cucurbitaceae & 20 & Leaves & Climber & Topical & M \\
\hline & & Amoebiasis & Momordica pterocarpa Hochst. ex A. Rich. & & & Leaves & Climber & Oral & \\
\hline 38 & Ounsi (M) & Stomach ache & Euclea divinorum Hiern & Ebenaceae & 26 & Root & Shrub & Oral & M \\
\hline \multirow[t]{2}{*}{39} & Sauto zao (M) & $\begin{array}{l}\text { Stomach bloating, food poisoning, } \\
\text { Vomiting }\end{array}$ & Acalypha fruticosa Forssk. & Euphorbiaceae & 98 & Leaves & Shrub & Oral & M \\
\hline & & Stomach ache & Acalypha fruticosa Forssk. & & & Stem & & Oral & M \\
\hline 40 & Sauti (M) & Eye injury & Acalypha volkensii Pax & Euphorbiaceae & 223 & Leaves & Herb & Topical & M \\
\hline 41 & No local name & Eye disease (Cataract) & Euphorbia hirta L. & Euphorbiaceae & 496 & Latex & Herb & Topical & M \\
\hline 42 & Tsedo (M) & Rabies & Euphorbia tirucalli L. & Euphorbiaceae & 285 & Root & Shrub & Oral & M \\
\hline 43 & Tsamo desho (M) & Wound & Ricinus communis L. & Euphorbiaceae & 350 & Seed & Shrub & Topical & $\mathrm{M}, \mathrm{A} 2$ \\
\hline
\end{tabular}


Table 1 Medicinal plants used in Maale and Ari communities with plant parts used, growth form and applications (Continued)

\begin{tabular}{|c|c|c|c|c|c|c|c|c|c|}
\hline 44 & Beto $(\mathrm{M})$ Beta $(\mathrm{A})$ & Gonorrhea ,Tape worm & $\begin{array}{l}\text { Croton macrostachyus } \\
\text { Hochst. ex Ferret \& Galinier }\end{array}$ & Euphorbiaceae & & Leaf bud & Tree & Oral & $\begin{array}{l}M, A 1, \\
A 2\end{array}$ \\
\hline \multirow[t]{2}{*}{45} & Gaina deshe/Azi & Stomach bloating, & Geranium arabicum Forssk. & Geraniaceae & 312,445 & Leaves & Herb & Oral & $\mathrm{A} 2$ \\
\hline & & Swelling & Geranium arabicum Forssk. & & & Leaves & Herb & Topical & \\
\hline 46 & Bato ketero deshe $(\mathrm{M})$ & Amoebiasis & $\begin{array}{l}\text { Pelargonium quinquelobatum } \\
\text { Hochst. ex A.Rich., }\end{array}$ & Geraniaceae & 227 & Whole & Herb & Oral & M \\
\hline 47 & Afi Deshe (A) & Evil eye & Hypericum peplidifolium A.Rich. & Hypericaceae & 263 & Leaves & Herb & Oral & $\mathrm{A} 1, \mathrm{~A} 2$ \\
\hline 48 & $\begin{array}{l}\text { Harsi deshe/ Gaina } \\
\text { deshe/Shai Shar (A) }\end{array}$ & Diarrhea & Ajuga leucantha Lukhoba & Lamiaceae & $274,278,441$ & Leaves & Herb & Oral & $\mathrm{A} 1, \mathrm{~A} 2$ \\
\hline 49 & Baye Apo Desho (M) & Stomach ache, Heart, Rheumatism & Becium filamentosum (Forssk.) Chiov. & Lamiaceae & $196,221,324$ & Whole & Herb & Oral & M \\
\hline \multirow[t]{2}{*}{50} & $\begin{array}{l}\text { Bokolo (M), } \\
\text { Dumfeken (A) }\end{array}$ & Stomach bloating, vomiting & Clerodendrum myricoides (Hochst.) Vatke & Lamiaceae & 195,346 & Leaves & Shrub & Oral & $\begin{array}{l}M, A 1, \\
A 2\end{array}$ \\
\hline & & Evil eye & $\begin{array}{l}\text { Clerodendrum myricoides } \\
\text { (Hochst.) Vatke }\end{array}$ & Lamiaceae & 38 & Root & Shrub & Oral ,Inhale & \\
\hline 51 & Apo Desho (M) & Evil eye & Endostemon tereticaulis (Poir.) M.Ashby & Lamiaceae & 99,165 & Whole & Herb & Topical & M \\
\hline 52 & Tsamo desho (M) & Cold & Fuerstia africana T.C.E. Fr. & Lamiaceae & 230 & Leaves & Herb & Topical & M \\
\hline \multirow[t]{2}{*}{53} & Pelo Tsala (M) & $\begin{array}{l}\text { Stomach ache, Amoebiasis, } \\
\text { Stomach bloating, Head ache, } \\
\text { Food poisoning, Vomiting }\end{array}$ & Leucas abyssinica (Benth.) Briq. & Lamiaceae & 167,321 & Leaves & Shrub & Oral & M \\
\hline & & Rheumantism & Leucas abyssinica (Benth.) Briq. & & & Leaves & & Topical & \\
\hline \multirow[t]{2}{*}{54} & Chergicola & $\begin{array}{l}\text { Pus from Ear, nose, mouth; } \\
\text { Eye disease (cataract), Rheumatism }\end{array}$ & Leucas glabarata (Nahl) Sm. in Rees & Lamiaceae & $\begin{array}{l}237,281,320 \\
365\end{array}$ & Leaves & Herb & Topical & M \\
\hline & & Headache & & & & Leaves & & Oral/inhale & \\
\hline 55 & Azi deshe & Swelling & Leucas martinicensis (Jacq.) R.Br. & Lamiaceae & 315 & Leaves & Herb & Topical & $A 2$ \\
\hline 56 & Lamo (M) & Stomach ache & Ocimum basilicum L. & Lamiaceae & 222,317 & Leaves & Herb & Oral & M \\
\hline 57 & Gurdarindo (M) & $\begin{array}{l}\text { Headache, Diarrhea Stomach } \\
\text { bloating, Stomach ache, Vomiting }\end{array}$ & Ocimum forskolei Benth. & Lamiaceae & 214,235 & Whole & Herb & Oral & M \\
\hline \multirow[t]{2}{*}{58} & Pasi kedo & Heart disease & Ocimum Ialiifolium Hochst. ex Benth. & Lamiaceae & 225,37 & Leaves & Herb & Oral & M, \\
\hline & Demakesse & Headache & Ocimum lamiifolium Hochst. ex Benth. & & 134 & Leaves & & Oral/Inhale & $\begin{array}{l}\mathrm{A} 1 \\
\mathrm{~A} 2\end{array}$ \\
\hline \multirow[t]{3}{*}{59} & Kuliti kup (M) & Herpes simplex ( "Mich") & Ocimum urticifolium Roth & Lamiaceae & 133 & Leaves & Shrub & Topical & $\mathrm{A} 1, \mathrm{~A} 2$ \\
\hline & & Stomach ache, Vomiting & Ocimum urticifolium Roth & & & & & Oral & \\
\hline & & Headache & Ocimum urticifolium Roth & & & & & Inhale & \\
\hline 60 & Pero (M) & Amoebiasis, Stomach ache & Plectranthus barbatus Andrews & Lamiaceae & 85 & Root & Herb & Oral & M \\
\hline 61 & Dumio (M) & Lower extremity weakness & $\begin{array}{l}\text { Plectranthus cylindraceus } \\
\text { Hochst. ex Benth. }\end{array}$ & Lamiaceae & 229,503 & Leaves & Herb & Topical & M \\
\hline
\end{tabular}


Table 1 Medicinal plants used in Maale and Ari communities with plant parts used, growth form and applications (Continued)

\begin{tabular}{|c|c|c|c|c|c|c|c|c|c|}
\hline 62 & $\begin{array}{l}\text { Ketero Desho } \\
\text { Solelo (M) }\end{array}$ & Stomach ache, Amoebiasis, Diarrhea & Plectranthus longipes Baker & Lamiaceae & 464 & whole & Herb & Oral & M \\
\hline 63 & Banjirindo (M) & Ease of Birth & Plectranthus punctatus (L.f) L.'Hér. & Lamiaceae & $207,318,369$ & Whole & Herb & Oral & M \\
\hline 64 & Anchip (M) & $\begin{array}{l}\text { Amoebiasis, Diarrhea, } \\
\text { Stomach bloating, Stomach } \\
\text { ache, Food poisoning }\end{array}$ & Pycnostachys abyssinica Fresen. & Lamiaceae & 205,319 & Leaves & Herb & Oral & M \\
\hline 65 & Sheto (M) & Constipation & Satureja abyssinica (Benth.) Briq. & Lamiaceae & 292,690 & Leaves & Herb & Oral & M \\
\hline 66 & $\begin{array}{l}\text { Zene gaime } \\
\text { Deshe }(A)\end{array}$ & Stomach ache & $\begin{array}{l}\text { Satureja paradoxa (Vatke) } \\
\text { Engl. ex Seybold }\end{array}$ & Lamiaceae & 310,371 & Leaves & Herb & Oral & $\mathrm{A} 2$ \\
\hline \multirow[t]{2}{*}{67} & Kayneka/Digita (A) & Stomach ache & Calpurnia aurea (Ait.) Benth. & Leguminosae & 247,272 & Leaves & shrub & Oral & $\begin{array}{l}\mathrm{A} 1 \\
\mathrm{~A} 2\end{array}$ \\
\hline & & Diarrhea & Calpurnia aurea (Ait.) Benth. & & & Root & Herb & Oral & \\
\hline 68 & Aro Dor deshe (M) & Malnutrition (Child) & Chamaecrista mimosoides (L.) Greene & Leguminosae & 197,322 & Whole & Herb & Oral & M \\
\hline \multirow[t]{2}{*}{69} & Dongordoso (M) & Tonsilites & Indigofera spicata Forssk. & Leguminosae & $\begin{array}{l}202,251 \\
256,261 \\
345,388\end{array}$ & Root & Herb & Oral & $M, A 1$ \\
\hline & $\begin{array}{l}\text { Afi Deshe/Gaina Deshe/ } \\
\text { Wesfat deshe (A) }\end{array}$ & $\begin{array}{l}\text { Diarrhea, Evil eye Ascariasis, } \\
\text { Stomach ache }\end{array}$ & Indigofera spicata Forssk. & & 467 & Whole & & Oral & \\
\hline 70 & Birbira (A) & To close wound caused by Jiggers & Millettia ferruginea (Hochst.) Baker & Leguminosae & - & Seed & Tree & Topical & $\mathrm{A} 2$ \\
\hline 71 & Dawrake (M) & Liver disease & $\begin{array}{l}\text { Piliostigma thonningii (Schumach.) } \\
\text { Milne-Redh. }\end{array}$ & Leguminosae & 117 & Leaves & Tree & Inhale & M \\
\hline 72 & Ara Deshe (A) & Liver disease (Ara) & Senna petersiana (Bolle) Lock & Leguminosae & 260 & Leaves & Shrub & Inhale & $\begin{array}{l}\mathrm{A} 1 \\
\mathrm{~A} 2\end{array}$ \\
\hline 73 & Karhaleko (M) & $\begin{array}{l}\text { Stomach ache, Diarrhea, Food } \\
\text { poisoning, Vomiting, Ascariasis }\end{array}$ & Senna singueana (Delile) Lock & Leguminosae & 59,28 & Root & Shrub & Oral & M \\
\hline \multirow[t]{2}{*}{74} & Dino desho $(M)$ & Diarrhea ( Children) & Stylosanthes fruticosa (Retz.) Alston & Leguminosae & & Leaves & Herb & Oral & M \\
\hline & & Snake bite & Stylosanthes fruticosa (Retz.) Alston & & & Root & & Oral & \\
\hline 75 & Dolkoiso (M) & Stomach ache, Vomiting & Tephrosia bracteolata Guill. \& Perr. & Leguminosae & 291 & Leaves & Shrub & Oral & M \\
\hline 76 & $\begin{array}{l}\text { Seringo demo } \\
\text { golodo }(M)\end{array}$ & Gastritis & Zornia pratensis Milne-Redh. & Leguminosae & 192,328 & Leaves & Herb & Oral & M \\
\hline 77 & Polo Golodo (M) & Stomach ache, Diarrhea, Gastritis & Abutilon longicuspe Hochst. ex A.Rich & Malvaceae & 193 & Leaves & Herb & Oral & M \\
\hline \multirow[t]{2}{*}{78} & Puta (M) & Heart, food poisoning & Gossypium herbaceum L. & Malvaceae & 621 & Leaves & Shrub & Oral & M \\
\hline & & Ear ache & Gossypium herbaceum L. & & & Leaf bud & & Topical & \\
\hline \multirow[t]{2}{*}{79} & $\begin{array}{l}\text { Wari Beshe }(M) \\
\text { Civil deshe }(A)\end{array}$ & $\begin{array}{l}\text { Oral trash, Diarrhea, Malnutrition } \\
\text { (Child) }\end{array}$ & $\begin{array}{l}\text { Kosteletzkya adoensis } \\
\text { (Hochst. ex A.Rich.) Mast. }\end{array}$ & Malvaceae & $209,368,465$ & Leaves & Herb & Oral & M, \\
\hline & & Fresh cut to stop bleeding & $\begin{array}{l}\text { Kosteletzkya adoensis } \\
\text { (Hochst. ex A.Rich.) Mast. }\end{array}$ & & & Leaves & Herb & Topical & $\mathrm{A} 1$ \\
\hline 80 & Chuksha (A) & Swelling & Sida rhombifolia L. & Malvaceae & 93,314 & Leaves & Herb & Topical & $\mathrm{A} 2$ \\
\hline
\end{tabular}

Sida rhombifolia L. 
Table 1 Medicinal plants used in Maale and Ari communities with plant parts used, growth form and applications (Continued)

\begin{tabular}{|c|c|c|c|c|c|c|c|c|c|}
\hline 81 & Kautso (M) & Vomiting, Food poisoning & Sterculia africana (Lour.) Fiori & Malvaceae & 47 & Leaves & Tree & Oral & M \\
\hline 82 & Gontersa (M) & Snake bite, Liver disease & Bersama abyssinica Fresen. & Melianthaceae & 436,475 & Stem bark & Shrub & Oral/Topical & $\mathrm{A} 1, \mathrm{~A} 2$ \\
\hline \multirow[t]{2}{*}{83} & Chorahe (M) & Amoebiasis & Chasmanthera dependens Hochst. & Menispermaceae & 100 & Stem & Herb & Oral & M \\
\hline & & Swelling & Chasmanthera dependens Hochst. & & & & & Topical & \\
\hline 84 & Balari (M) & $\begin{array}{l}\text { Amoebiasis, Diarrhea, Rabies, } \\
\text { Stomach ache }\end{array}$ & Cissampelos mucronata A.Rich. & Menispermaceae & 29,96 & Root & Herb & Oral & M \\
\hline \multirow[t]{2}{*}{85} & Haleko, Kellengi (A) & Eye disease (cataract) & Moringa stenopetala (Bak.) Cuf. & Moringaceae & - & Stem bark & Tree & Topical & M, \\
\hline & & Malaria & Moringa stenopetala (Bak.) Cuf. & & & Leaves & Tree & Oral & $\mathrm{A} 1$ \\
\hline 86 & Musi (A) & Diarrhea & Musa paradisiaca L. & Musaceae & - & Fruit & Herb & Oral & A1 \\
\hline 87 & Enkoko (A) & Tape worm & Embelia schimperi Vatke & Myrsinaceae & - & Seeds & Shrub & Oral & $\mathrm{A} 2$ \\
\hline 88 & Diko (M) & $\begin{array}{l}\text { Lower extremity weakness, } \\
\text { Rheumatism }\end{array}$ & $\begin{array}{l}\text { Commicarpus grandiflorus } \\
\text { (A.Rich) Standley }\end{array}$ & Nyctaginaceae & & Leaves & Herb & Topical & M \\
\hline 89 & Mukalle (M) & Wound & Ximenia caffra Sond. & Olacaceae & 22 & Seed & Tree & Topical & M \\
\hline 90 & Chamo (M) & Tape worm & $\begin{array}{l}\text { Jasminum grandiflorum } L \text { subsp. } \\
\text { floribundum (R.Br. ex Fresen.) P.S.Green: }\end{array}$ & Oleaceae & 415,727 & & Climber & Oral & M \\
\hline 91 & Rimiti (M) & Ascariasis, Gonorrhea & $\begin{array}{l}\text { Olea europaea L. subsp. cuspidata } \\
\text { (Wall.ex G.Don) Cif. }\end{array}$ & Oleaceae & 108 & Leaves & Tree & Oral & M \\
\hline 92 & $\begin{array}{l}\text { Afi deshe } \\
\text { (Bere Keno) (A) }\end{array}$ & Evil eye & Biophytum umbraculum Welw. & Oxalidaceae & 264 & Leaves & Herb & Oral & $\mathrm{A} 2$ \\
\hline 93 & $\begin{array}{l}\text { Solcarindo }(M) \text {, } \\
\text { Kinsa kins }(A)\end{array}$ & $\begin{array}{l}\text { Malnutrition (children) Diarrhea, } \\
\text { Tooth ache, Stop fresh cut bleeding }\end{array}$ & Oxalis radicosa A. Rich. & Oxalidaceae & 212,326 & $\begin{array}{l}\text { Leaves with } \\
\text { succulent stem }\end{array}$ & Herb & Oral & $M, A 1$ \\
\hline 94 & Azi (ite) deshe & Swelling & Phyllanthus ovalifolius Forssk. & Phyllanthaceae & 200 & Leaves & Shrub & Topical & $\mathrm{A} 1, \mathrm{~A} 2$ \\
\hline 95 & Afi Deshe (A) & Evil eye & Phyllanthus rotundifolius Willd & Phyllanthaceae & 244,305 & Leaves & Herb & Oral & A1 \\
\hline 96 & Tolsi (M), Andod (A) & Gonorrhea, Stomach bloating & Phytolacca dodecandra L 'Hérit. & Phytolaccaceae & 97,241 & Leaves & Climber & Oral & $\mathrm{M}, \mathrm{A} 1$ \\
\hline \multirow[t]{2}{*}{97} & $\begin{array}{l}\text { Kurupe }(M) \text {, } \\
\text { Guni deshe }(A)\end{array}$ & Tooth ache & Plumbago zeylanica $\mathrm{L}$. & Plumbaginaceae & $\begin{array}{l}90,279 \\
284,306,367\end{array}$ & Root bark & Climber & Topical & $\begin{array}{l}M, A 1 \\
A 2\end{array}$ \\
\hline & & Snake bite & Plumbago zeylanica L. & & & Whole & & Oral & \\
\hline 98 & Tsoralle (M) & Skin burns & Portulaca quadrifida $\mathrm{L}$. & Portulacaceae & & Leaves & Herb & Topical & M \\
\hline 99 & Wuchanbe (M) & Tape worm & Myrsine africana $\mathrm{L}$. & Primulaceae & 178 & Seed & Shrub & Oral & M \\
\hline 100 & Gero (M) & Tooth ache & Faurea speciosa Welw. & Proteaceae & 109,413 & Leaves & Tree & Topical & M \\
\hline \multirow[t]{2}{*}{101} & Dishoo (M) & Ear ache & Clematis hirsuta Perr. \& Guill. & Ranunculaceae & 110,231 & Leaves & Climber & Topical & M \\
\hline & & Headache & Clematis hirsuta Perr. \& Guill. & & & Leaves & & Oral & \\
\hline \multirow[t]{2}{*}{102} & Afi Deshe (M) & Evil eye & Ranunculus multifidus Forssk. & Ranunculaceae & $381,111,270$ & Leaves & Herb & Topical & $A 2$ \\
\hline & & Tonsillitis & Ranunculus multifidus Forssk. & & & & & Oral & \\
\hline 103 & Ziambee & Ascariasis & $\begin{array}{l}\text { Caylusea abyssinica (Fresen.) } \\
\text { Fisch. \& Mey }\end{array}$ & Resedaceae & 16 & Leaves & Herb & Oral & M \\
\hline
\end{tabular}


Table 1 Medicinal plants used in Maale and Ari communities with plant parts used, growth form and applications (Continued)

\begin{tabular}{|c|c|c|c|c|c|c|c|c|c|}
\hline 104 & Kulmi (A) & Tonsilities & Rhamnus prinoides L.'Hérit. & Rhamnaceae & - & Leaves & Shrub & Oral & $\mathrm{A} 1, \mathrm{~A} 2$ \\
\hline 105 & Kosso (A) & Tape worm & Hagenia abyssinica (Brace) J.F.Gmel. & Rosaceae & - & Flower & Tree & Oral & $M, A 2$ \\
\hline 106 & Afi Deshe(b) & Evil eye & Oldenlandia lancifolia (Schumach.) DC. & Rubiaceae & 245 & Leaves & Herb & Oral & A1 \\
\hline \multirow[t]{2}{*}{107} & Wari ampi (M) & Liver disease (Ara) & Pavetta gardeniifolia A.Rich. & Rubiaceae & 325 & Leaves & Shrub & Inhale & M \\
\hline & & Common cold & Pavetta gardeniifolia A Rich. & & & & & & \\
\hline 108 & $\begin{array}{l}\text { Afi deshe/gaina deshe } \\
\text { (A) }\end{array}$ & $\begin{array}{l}\text { Diarrhea, Evil eye, Tooth ache, } \\
\text { Stomach ache, Head wound }\end{array}$ & Pentas lanceolata (Forssk.) Deflers & Rubiaceae & $\begin{array}{l}277,243,250 \\
276,249\end{array}$ & Root & Herb & Oral & $\begin{array}{l}\mathrm{A} 1 \\
\mathrm{~A} 2\end{array}$ \\
\hline 109 & Garo (M) & Ascariasis & Vangueria apiculata K. Schum. & Rubiaceae & 8,157 & Leaves & Shrub & Oral & M \\
\hline 110 & Gembala (M) & Malaria & Gardenia ternifolia Schumach. \& Thonn. & Rubiaceae & 352 & Leaves & Tree & Oral & M \\
\hline 111 & Onaki (M) & Ascariasis & $\begin{array}{l}\text { Meyna tetraphylla (Schweinf. } \\
\text { ex Hiern) Robyns }\end{array}$ & Rubiaceae & 4 & Leaves & Tree & Oral & M \\
\hline 112 & Lomi (A) & Oral trash; Food poisoning & Citrus aurantiifolia (Christm.) Swingle & Rutaceae & - & Fruit & Tree & Oral & A1 \\
\hline 113 & Tselto (M) & Stomach ache, common cold & Ruta chalepensis L. var.tenuifolia D'Urville & Rutaceae & - & $\begin{array}{l}\text { Leaves with } \\
\text { succulent stem }\end{array}$ & Herb & Oral & $\begin{array}{l}M, A 1 . \\
A 2\end{array}$ \\
\hline 114 & Gedai (M) & Common cold & Zanthoxylum chalybeum Engl. & Rutaceae & 31 & Seed & Tree & Oral & M \\
\hline 115 & Wulchi (M) & Scorpion bite & Anemia schimperiana Presl. & Schizaeaceae & 290 & Leaves & Herb & Oral & M \\
\hline 116 & Mitmita (A) & Malaria & Capsicum annuum L. & Solanaceae & - & Fruit & Herb & Oral & A1 \\
\hline 117 & Guni deshe (A) & Snake bite & Datura metel L. & Solanaceae & 103 & Whole & Herb & Oral/topical & $\mathrm{A} 1$ \\
\hline 118 & Atsefaris (M) & Toothache & Datura stramonium L. & Solanaceae & & Leaf bud & Shrub & Topical & M \\
\hline 119 & Ara Deshe $(A)$ & Liver disease & Discopodium penninervium Hochst. & Solanaceae & 339 & Leaves & Tree & Inhale & $\mathrm{A} 2$ \\
\hline 120 & Tumbaho (M) & Leeches & Nicotiana tabacum L. & Solanaceae & - & Leaves & Herb & $\begin{array}{l}\text { Topical/ } \\
\text { Oral }\end{array}$ & $\mathrm{A} 1, \mathrm{~A} 2$ \\
\hline 121 & $\begin{array}{l}\text { Achi Kolpo (M), Garenti } \\
\text { (A) }\end{array}$ & Amoebiasis, Stomach ache, Evil eye & Solanum dasyphyllum Schumach. & Solanaceae & 9,94 & Root & Herb & Oral & $\begin{array}{l}M, A 1 \\
A 2\end{array}$ \\
\hline 122 & $\begin{array}{l}\text { Kotse Garenti (A) } \\
\text { Bulabulo(M) }\end{array}$ & Ascariasis, stomach ache & Solanum incanum L. & Solanaceae & 94 & Root & Herb & Oral & M \\
\hline 123 & Muto (M) & Cold & Withania somnifera (L.) Dunal & Solanaceae & 176,208 & Root & Herb & Oral & M \\
\hline 124 & Azi deshe/masna (A) & Swelling & Veronica abyssinica Fres. & Scrophulariaceae & 316,334 & Leaves & Herb & Topical & $\mathrm{A} 2$ \\
\hline 125 & Enaro (M) & Headache, Vomiting & Lantana camara L. & Verbenaceae & 169 & Leaves & Shrub & Oral & M \\
\hline 126 & Dolo amede (M) & Headache & Lantana trifolia L. & Verbenaceae & 190 & Leaves & Shrub & Oral & M \\
\hline 127 & Atuch & Stomach ache & $\begin{array}{l}\text { Verbena officinalis subsp. } \\
\text { africana R.Fernandes \& Verdc. }\end{array}$ & Verbenaceae & 268 & Leaves & Herb & Oral & $\mathrm{A} 2$ \\
\hline 128 & Kuze (M) & Ascariasis, Food poisoning ,Vomiting & $\begin{array}{l}\text { Balanites rotundifolia } \\
\text { (van Tieghem) Blatter }\end{array}$ & Zygophyllaceae & 41 & Leaves & Tree & Oral & M \\
\hline
\end{tabular}

Study sites: $M=$ Maale, $A 1=$ Ari 1and $A 2=$ Ari 2 ; Plant names are checked based on www.theplantlist.org. 
Table 2 Average number of medicinal plants cited per respondent groups

\begin{tabular}{|c|c|c|c|c|c|c|}
\hline \multirow{2}{*}{ Study sites } & \multicolumn{2}{|c|}{ Gender } & \multirow{2}{*}{$\begin{array}{c}\text { T-test } \\
\text { P-value }\end{array}$} & \multicolumn{2}{|c|}{ Age groups } & \multirow{2}{*}{$\begin{array}{l}\text { T-test } \\
\text { P-value }\end{array}$} \\
\hline & Male & Female & & $>40$ & $\leq 40$ & \\
\hline Maale $(n=26)$ & 20.33 & 13.64 & $0.01^{*}$ & 19.67 & 12.73 & $0.01^{*}$ \\
\hline Ari $1(n=24)$ & 9.40 & 5.00 & $0.00^{*}$ & 8.06 & 7.22 & 0.65 \\
\hline Ari $2(n=24)$ & 7.57 & 3.80 & $0.00^{*}$ & 6.77 & 5.09 & 0.13 \\
\hline Overall & & & $0.01^{*}$ & & & \\
\hline
\end{tabular}

People aged above 40 are considered matured adult by the community;

P-values $<0.05$ are marked with*

relative lack of knowledge in the young will further be aggravated in the future when many species become scarce in the landscape and this might have negative impact on knowledge continuity in the near future. On the other hand, the results from Ari showed that knowledge is not always disappearing, as there were no significant differences in plant knowledge between age groups.

Table 3 shows the familiarity indices of medicinal plants for the treatment of different types of health problems. Meyna tetraphylla in Maale and Solanum dasyphyllum in Ari 1 and 2 were most cited. There was little correspondence between the two sites with regard to frequently mentioned plant species, but health problems treated with medicinal plants were quite similar. Species with high familiarity indices should be considered for further phytochemical and pharmacological studies.

Preference ranking among ten key informants for eight selected medicinal plants used for the treatment of ascariasis is shown in Table 4. From Table 4 it appears that people had certain preferences for medicinal plants based on their perceived efficacy for the treatment of the frequently cited health problem, ascariasis. Species with higher preference ranking may indicate effective healing properties, which suggests that they are interesting for further phytochemical and pharmacological research.

\section{Mechanisms of knowledge transfer among social groups}

Most medicinal plant knowledge is transferred orally, as was reported by 71 study participants (96\%) in the study sites (Table 5). This is the dominant mechanism of traditional knowledge transfer system in Africa [8], although this type of transfer cannot guarantee continuity under the current circumstances, where plant resource degradation and loss is severe. Most people (82\%) obtained their knowledge from their (grand) parents, which is similar to the percentage found by a study in Wonago district, Ethiopia [41]. The great majority of the study participants preferred to transfer their medicinal plant knowledge to their children or grandchildren, which favours knowledge conservation and continuity mostly within the family line.
The majority of study participants who showed interest to transfer their medicinal plant knowledge, preferred to transfer this to their first son. This preference was associated with the perception and fear that daughters would share the knowledge with their husbands' family when they get married. Knowledge transfer to the new family was not appreciated by respondents with the perception that their secret knowledge would be known by others. When the first son was not considered trustworthy to keep the knowledge secretly or not judged interested in the subject as assessed through what he says, what he does and his general attitudes, parents transferred it to their second son or grandson.

In most cases there existed a concern among communities and elder knowledgeable people on plant resource degradation around their village. Dwindling resources around settlements may have negative implications for the future transfer of medicinal plant knowledge, as elders are unable to walk long distances from their residence. If the plants are no longer available, it becomes difficult to show and teach others about their names, characteristics and uses.

\section{Demographic factors influencing medicinal plant knowledge} Gender significantly predicted medicinal plant knowledge. Male study participants knew a higher number of medicinal plants than female ones. This is probably associated with the perception and culture of both ethnic communities to favour males in transferring medicinal plant knowledge. This must have implications for the cultivation of medicinal plant species in home gardens as women play a major role in managing these gardens. Moreover, site also strongly influenced the number of medicinal plants known and used (Table 6). Religion, family size and education did not influence plant knowledge.

Older members of the community in Maale knew more medicinal plants than youngsters (Table 2), which may also reflect an ongoing gradual knowledge loss of knowledge in the study community. Hence, it is important to include traditional knowledge in the school curricula to raise awareness as recommended by Awas [11].

\section{Similarities on in medicinal plant knowledge among sites} Generally we found little similarity among the three study sites. The calculated Jaccard similarity index was relatively higher between the two Ari sites (0.33), lower between Maale and Ari 1 (0.14) and lowest between Maale and Ari 2 (0.08). Our results showed that the Ari 1 and Ari 2 sites are more similar in medicinal plant knowledge than each of them compared to Maale. This can be explained by the geographical proximity between the two Ari sites and also supported by the fact that they belong to the same ethnic group and share their cultural background. 
Table 3 Familiarity index (FI) of medicinal plants In Maale (M), Ari 1 (A1) and Ari 2 (A2)

\begin{tabular}{|c|c|c|c|c|c|}
\hline Scientific name & Family name & Illness & Frequency & $\mathrm{FI}$ & Site \\
\hline Meyna tetraphylla & Rubiaceae & Ascariasis & 11 & 0.42 & M \\
\hline Plectranthus barbatus & Lamiaceae & Amoebiasis & 11 & 0.42 & M \\
\hline Ozoroa insignis & Anacardiaceae & Ascariasis & 11 & 0.42 & M \\
\hline Hypoestes forskaolii & Acanthaceae & Stomach ache & 10 & 0.38 & M \\
\hline Ocimum basilicum & Lamiaceae & Stomach ache & 10 & 0.38 & M \\
\hline Celosia trigyna & Amaranthaceae & Tapeworm & 10 & 0.38 & M \\
\hline Plectranthus barbatus & Lamiaceae & Stomach ache & 10 & 0.38 & M \\
\hline Solanum dasyphyllum & Solanaceae & Stomach ache & 16 & 0.67 & $\mathrm{~A} 1$ \\
\hline Indigofera spicata & Leguminosae & Ascariasis & 12 & 0.50 & $\mathrm{~A} 1$ \\
\hline Ruta chalepensis & Rutaceae & Stomach ache & 12 & 0.50 & $\mathrm{~A} 1$ \\
\hline Plumbago zeylanica & Plumbaginaceae & Snake bite & 10 & 0.42 & $\mathrm{~A} 1$ \\
\hline Acmella caulirhiza & Compositae & Tonsillitis & 9 & 0.38 & $\mathrm{~A} 1$ \\
\hline Kosteletzkya adoensis & Malvaceae & Fresh cut to stop bleeding & 9 & 0.38 & A1 \\
\hline Vernonia amygdalina & Compositae & Malaria & 9 & 0.38 & $\mathrm{~A} 1$ \\
\hline Citrus aurantifolia & Rutaceae & Oral trash & 9 & 0.38 & A1 \\
\hline Acmella caulirhiza & Compositae & Tooth ache & 8 & 0.33 & $\mathrm{~A} 1$ \\
\hline Oxalis radicosa & Oxalidaceae & Fresh cut to stop bleeding & 8 & 0.33 & $\mathrm{~A} 1$ \\
\hline Rhamnus prinoides & Rhamnaceae & Tonsillitis & 8 & 0.33 & $\mathrm{~A} 1$ \\
\hline Citrus aurantifolia & Rutaceae & Food poisoning & 8 & 0.33 & $\mathrm{~A} 1$ \\
\hline Solanum dasyphyllum & Solanaceae & Stomach ache & 10 & 0.42 & $A 2$ \\
\hline Garcinia livingstonei & Clusiaceae & Oral trash & 10 & 0.42 & A2 \\
\hline Millettia ferruginea & Leguminosae & Close wound caused by Jiggers & 9 & 0.38 & $A 2$ \\
\hline Nicotiana tabacum & Solanaceae & Leeches & 8 & 0.33 & A2 \\
\hline Hagenia abyssinica & Rosaceae & Tape worm & 8 & 0.33 & $\mathrm{~A} 2$ \\
\hline
\end{tabular}

\section{Medicinal plant collection, conservation efforts and} major threats

Study participants mostly collected medicinal plants from crop fields, home gardens and nearby forest patches. The results of the growth form analysis revealed that herbs were the most common growth form and the dominant plant parts harvested were leaves. This was found by

Table 4 Preference ranking of eight medicinal plants used for the treatment of ascariasis based on perceived efficacy by ten respondents in Maale

\begin{tabular}{lllll}
\hline Scientific name & $\begin{array}{l}\text { Total sum of } \\
\text { ranks }(\mathbf{n = 1 0})\end{array}$ & $\begin{array}{l}\text { Standard } \\
\text { deviation }\end{array}$ & $\begin{array}{l}\text { Mean } \\
\text { ranking }\end{array}$ & $\begin{array}{l}\text { Rank } \\
\text { values }\end{array}$ \\
\hline Ozoroa insignis & 18 & \pm 1.0 & 1.8 & 1 \\
Meyna tetraphylla & 22 & \pm 1.4 & 2.2 & 2 \\
Indigofera spicata & 34 & \pm 1.6 & 3.4 & 3 \\
Vangueria apiculata & 45 & \pm 1.6 & 4.5 & 4 \\
Balanites rotundifolia & 54 & \pm 2.1 & 5.4 & 5 \\
Pergularia daemia & 59 & \pm 1.7 & 5.9 & 6 \\
Senna singueana & 61 & \pm 0.6 & 6.1 & 7 \\
Combretum aculeatum & 67 & \pm 2.0 & 6.7 & 8 \\
\hline
\end{tabular}

many researchers in different parts of East Africa [42,43]. Harvesting of leaves may not have negative effects on resource availability, provided that the plant itself is not destroyed during harvesting, which is especially relevant for herbs.

A few study participants brought seedlings of medicinal herbs from fields and forest patches and started cultivating them in their home gardens. Their main reasons for doing so were to conserve plants that were scarce in their surroundings, to keep herbs available that were unavailable during the dry season and to have the medicine at hand during emergency situations. The practice of nurturing of wild species in home gardens was limited to a very small area, because people suspected that their importance as medicine could be easily guessed by outsiders. This unwillingness to share resources with neighbours or other non-family members may negatively affect the conservation of medicinal plant knowledge and resources. Giday and Teklehaymanot [44] did not encounter any cultivation of medicinal plants by Afar people in Ethiopian Ada'ar district. The main reason mentioned for this was the easily availability of the medicinal plant in areas that are not far from the homesteads. 
Table 5 Acquisition and willingness to transfer medicinal plant knowledge in the study sites

\begin{tabular}{lllll}
\hline & Maale frequency (\%) & Ari 1 frequency (\%) & Ari 2 frequency (\%) & Total frequency (\%) \\
\hline $\begin{array}{llll}\text { Knowledge acquired } \\
\text { Parents/grandparents }\end{array}$ & $20(76.9)$ & $19(79.1)$ & & \\
Friends & $1(3.9)$ & $1(4.2)$ & $0(91.7)$ & $2(2.7)$ \\
Neighbours & $2(7.7)$ & $1(4.2)$ & $0(0.0)$ & $3(4.1)$ \\
$\begin{array}{l}\text { Other (Given from God, } \\
\text { accidentally encountered individuals) }\end{array}$ & $3(11.5)$ & $3(12.5)$ & $2(8.3)$ & $8(10.8)$ \\
Knowledge willing to transfer to: & & & & $59(79.7)$ \\
Children and grand children & $17(65.4)$ & $22(91.6)$ & $20(87.5)$ & $6(8.1)$ \\
Any family member & $4(15.4)$ & $1(4.2)$ & $1(4.2)$ & $4(5.4)$ \\
Neighbours & $4(15.4)$ & $0(0.0)$ & $3(0.0)$ & $5(6.8)$ \\
No one & $1(3.8)$ & $1(4.2)$ & $3(12.5)$ & \\
\hline
\end{tabular}

Agricultural expansion and lack of cultivation tradition were indicated by our study participants as major threats in all study sites (Table 7). The conversion of natural vegetation to agricultural fields is a serious issue in Eastern Africa, where the rural population is highly dependent on subsistence agriculture [45]. Sustainable management of resources may not be an easy task, but it is crucial to guarantee future access to herbal medicine for rural communities [46]. Other threats that were mentioned were a lack of maintenance and the fact that people with little knowledge considered medicinal plants growing among their crops as weeds were likely to uproot them.

Most respondents (76\% in Maale, $75 \%$ in Ari 1, 83\% in Ari 2) collected plants at any time or day of the week. A few herbalists did not harvest plants on Sundays and some orthodox Christians did not harvest on Wednesdays and

Table 6 Socio-demographic and site factors prediction on the number of medicinal plant knowledge

\begin{tabular}{lcccc}
\hline & \multicolumn{2}{c}{ Unstandardized } & Standardized & \\
\cline { 2 - 3 } & $\begin{array}{c}\text { Beta } \\
\text { coefficient }\end{array}$ & $\begin{array}{c}\text { Std. } \\
\text { error }\end{array}$ & $\begin{array}{c}\text { Beta } \\
\text { coefficient }\end{array}$ & p-value \\
\hline$R^{2}=74.2$ & 3.831 & 0.443 & & \\
Constant & 0.016 & 0.006 & 0.198 & $0.014^{*}$ \\
Age & -0.819 & 0.149 & -0.419 & $0.000^{*}$ \\
Gender & 0.266 & 0.167 & 0.135 & 0.117 \\
Educational level & -0.041 & 0.025 & -0.122 & 0.101 \\
Family size & -1.485 & 0.185 & -0.716 & $0.000^{*}$ \\
Site (Ari 1) & -1.905 & 0.189 & -0.918 & $0.000^{*}$ \\
Site (Ari 2) & 0.136 & 0.178 & 0.068 & 0.449 \\
Religion & & & & \\
(Protestant Christian) & 0.190 & 0.199 & 0.093 & 0.343 \\
Religion & & & & \\
(Orthodox Christian) & & & & \\
\hline Stes are compared against & & & & \\
\end{tabular}

Sites are compared against Maale site. Religion is compared against traditional religion. Variables with a significant influence $(P<0.05)$ are marked with*.
Fridays as these are fasting days. In emergency cases, they used stored herbal medicine on these days.

\section{Commercialization of herbal medicine}

Marketing of medicinal plants was not common at the studied markets, apart from the well-known Hagenia abyssinica flowers and Embelia shimperi seeds (both wild collected) and the cultivated Allium sativum and Artemisia absinthium that are also used as spices. The commercialization of other wild and semi-wild species is hampered by the fact that medicinal knowledge is only held by few people. Tolassa [21] also found only a few species (Thalictrum rhynchocarpum, Piper capense and Echinops kebericho) at Gimibi and Gaba Senbeta markets, in western Ethiopia, while Giday et al. [16] found that the few species sold by Bench communities in south-western Ethiopia doubled as spices. In our study area, market chains were short and medicinal plants were directly sold by harvesters without further processing. The economic importance of the trade was limited: the price of Embelia shimperii seeds was only 2 Ethiopian Birr $(0.10$ \$) per glass (about $250 \mathrm{ml}$ ). The product was not always available and marketed in small quantities.

Although herbalists' incomes obtained through giving treatments to local communities were not high, the most important aspect observed from traditional healers is local recognition and respect by the community. In our study sites, respondents mentioned that on average they were consulted by patients five times per month. The charges for a treatment depended on the type of health problem treated and on patient/healers' relationships. Payment per treatment ranged from 1-10 Ethiopian Birr (equivalent to $0.05-0.5 \$$ ) and sometimes were free of charge, especially in Maale area. However, in the Ari sites traditional healers believed that whatever relation existed, the patient had to pay money for a consult; otherwise they underlined that the medicine would not be effective. Limited income obtained from marketing of 
Table 7 Threats for medicinal plant resources and their priority ranking

\begin{tabular}{|c|c|c|c|c|c|c|}
\hline \multirow[b]{2}{*}{ Threats } & \multicolumn{3}{|c|}{ Herbs } & \multicolumn{3}{|c|}{ Trees, shrubs, climbers } \\
\hline & Maale & Ari 1 & Ari 2 & Maale & Ari 1 & Ari 2 \\
\hline Agricultural land expansion & 2 & 2 & 2 & 1 & 1 & 1 \\
\hline Lack of cultivation and maintenance & 1 & 1 & 1 & 2 & 2 & 2 \\
\hline Conflicting uses (timber, fencing, firewood, etc) & 0 & 0 & 0 & 3 & 3 & 3 \\
\hline Grazing pressure & 4 & 4 & 4 & 4 & 5 & 4 \\
\hline Drought or unreliable rainfall & 3 & 3 & 3 & 5 & 4 & 5 \\
\hline
\end{tabular}

"0"' $=$ not mentioned as a threat.

medicinal plants or from treatments given to patients may have negative implications future cultivation, maintenance and conservation of medicinal plants in the landscape.

\section{Conclusion}

This study indicated that medicinal plants were important for the health care of the Maale and Ari communities, as they used at least 128 species and traditional medicine was considered as the first line of treatment by $89 \%$ of our respondents. Knowledge differed between and within ethnic groups and also among sites. The fact that knowledge transfer was predominantly to family members and in particular to first-born sons may negatively affect its continuity and may result in knowledge loss if medicinal plant resources become scarce in the future. Low income obtained from marketing of medicinal plants and herbal treatments may have strong implications on the future conservation of medicinal plants in the landscape.

Agricultural land expansion and a lack of cultivation practices limit the availability of medicinal plant resources in the area. Urgent action is required towards conservation (both ex-situ and in-situ combined) of medicinal plants and traditional knowledge before we lose them in the near future. Moreover, land use planning and development plan should also consider strategies that stimulate medicinal plant availability in the landscape and work towards increasing their cultivation to complement exand in-situ conservation efforts.

Popular medicinal species such as Solanum dasyphyllum, Indigofera spicata, Plumbago zeylanica, Meyna tetraphylla and multi-use species like Oxalis radicosa are good candidates for consideration in further phytochemical and pharmacological research to verify their efficacy.

\section{Competing interests}

The authors declare that they have no competing interests.

\section{Authors' contributions}

The first author collected and analyzed the data, and wrote the draft manuscript and other authors made comments on the data analysis and on the write-up of the manuscript. All authors read and approved the final manuscript.

\section{Acknowledgements}

This research project was granted by the Netherlands Organization for International Cooperation in Higher Education (Netherlands Fellowship Programme) as part of the PhD research of the first author. Our gratefulness goes to the informants in all study sites who shared with us their knowledge on medicinal plants used for the treatment of human health problems. Our appreciation goes also to the staff of the National Herbarium of Addis Ababa University and the Biosystematics group at Wageningen University for their technical assistance. We are also thankful to zonal, district and kebele administrators and experts (health, forestry and agricultural development) in the study area.

\section{Author details}

${ }^{1}$ Ethiopian Institute of Agricultural Research, Forestry Research Center, P.O. Box 58532, Addis Ababa, Ethiopia. ${ }^{2}$ Biosystematics Group, Wageningen University and Research Center, P.O. Box 647, 6700 Wageningen, AP, The Netherlands. ${ }^{3}$ Naturalis Biodiversity Center, Leiden University, P.O. Box 9517 , 2300 Leiden, RA, The Netherlands. ${ }^{4}$ Department of Plant Biology and Biodiversity Management, The National Herbarium, Addis Ababa University, P.O. Box 3434, Addis Ababa, Ethiopia.

Received: 26 November 2013 Accepted: 30 April 2014

Published: 4 June 2014

\section{References}

1. Chaya N: Poor access to health Services: Ways Ethiopia is overcoming it. Res Comment 2007, 2(2):1-6.

2. IBC: National biodiversity strategy and action plan. Addis Ababa, Ethiopia: Government of the federal Democratic Republic of Ethiopia; 2005:103.

3. Flatie T, Gedif T, Asres K, Gebre-Mariam T: Ethnomedical survey of Berta ethnic group Assosa Zone, Benishangul-Gumuz regional state, mid-west Ethiopia. J Ethnobiol Ethnomed 2009, 5(14):1-11.

4. Gedif T, Hahn HJ: The use of medicinal plants in self-care in rural central Ethiopia. J Ethnopharmacol 2003, 87:155-161.

5. Yirga G, Zeraburk S: Ethnobotanical study of traditional medicinal plants of Gindeberet district, Western Ethiopia. Mediterr J Soc Sci 2011, 2(4):49-54.

6. Joy PP, Thomas J, Mathew S, Skaria BP: Medicinal Plants. In Trop Hort. 2nd edition. Edited by Bose TK, Kabir J, Das P, Joy PP. Calcutta: Naya Prokash; 2001:449-632.

7. Bekele E: Study on Actual Situation of Medicinal Plants in Ethiopia, Addis Ababa. 2007.

8. Fassil H: We do what we know: local health knowledge and home-based medicinal plant use in Ethiopia. PhD thesis Green College, Oxford University; 2003.

9. Fassil H: Beyond Plants, Professionals \& Parchments: The role of home-based medicinal plant use and traditional health knowledge in primary health care in Ethiopia. 2005

10. Giday M: Medicinal plant of the Bench, Meinit and Sheko cultural groups in Ethiopia. Addis Ababa, Ethiopia: PhD Thesis, Addis Ababa University; 2007.

11. Awas T: Plant diversity in Western Ethiopia, Ecology, Ethnobotany and conservation. Norway: PhD thesis, University of Oslo; 2007.

12. Regassa T: State constitutions in Federal Ethiopia: A preliminary observation: a summary for the Bellagio Conference. 2004:1-12.

13. Hunde D, Asfaw Z, Kelbessa E: Use of traditional medicinal plants by people of 'Boosat' sub district, central eastern Ethiopia. Ethiop J Health Sci 2006, 16(2):141-155.

14. Teklehaymanot T, Giday M: Ethnobotanical study of medicinal plants used by people in Zegie Peninsula, North-western Ethiopia. J Ethnobiol Ethnomed 2007, 3(12):1-11.

15. Yineger $H$, Yewhalaw D, Teketay D: Ethnomedicinal plant knowledge and practice of the Oromo ethnic group in south-western Ethiopia. J Ethnobiol Ethnomed 2008, 4(1):1-10. 
16. Giday M, Asfaw Z, Woldu Z, Teklehaymanot T: Medicinal plant knowledge of the Bench ethnic group of Ethiopia: an ethnobotanical investigation. J Ethnobiol Ethnomed 2009, 5(34):1-10

17. Teklehaymanot T: Ethnobotanical study of knowledge and medicinal plants use by people in Dek Island in Ethiopia. J Ethnopharmacol 2009, 124:69-78.

18. Ashagre M: Ethnobotanical Study of Medicinal Plants in Guji Agro-pastoralists. Blue Hora District of Borana Zone: Oromia Region, Ethiopia. MSc thesis. Addis Ababa University; 2011.

19. Dalle GT, Maass BL, Isselstein J: Plant biodiversity and ethnobotany of pastoralists in southern Oromia. Ethiop Econ Bot 2005, 59(1):43-65.

20. Yirga G: Assessment of indigenous knowledge of medicinal plants in Central zone of Tigray, Northern Ethiopia. Afr J Plant Sci 2010, 4:006-011.

21. Tolasa E: Use and conservation of traditional medicinal plants by indigenous people in Gimbi woreda, western Wellega, Ethiopia. MSc thesis. Addis Ababa University; 2007:121.

22. Friis Bl, Demissew S, Van Breugel P: Atlas of the Potential Vegetation of Ethiopia. Biologiske Skrifter (Biol.Skr.Dan.Vid.Selsk.) 2010, 58:1-307.

23. CSA: Summary and Statistical Report of the 2007 Population and Housing Censes. 2008

24. Martin GJ: Ethnobotany: a methods manual London. UK: Chapman and Hall; 1995.

25. Alexiades M: Collecting ethnobotanical data. An introduction to basic concepts and techniques. In Selected Guideline for ethnobotanical research: A Field Manual. Edited by Alexiades M. U.S.A. Sheldon JW: The New York Botanical Garden; 1996:53-94

26. Cotton CM: Ethnobotany: Principles and applications Chichester. New York: John Wiley and Sons Ltd; 1996.

27. Edwards S, Tadesse M, Hedberg I (Eds): Canellaceae to Euphorbiaceous. Flora of Ethiopia and Eritrea Volume 2. Addis Ababa, Ethiopia: Issue part 2. The National Herbarium; 1995. Department of Systematic Botany, Uppsala, Sweden.

28. Edwards S, Demissew S, Hedberg I (Eds): Hydrocharitaceae to Arecaceae. Flora of Ethiopia and Eritrea Volume 6. Addis Ababa, Ethiopia: The National Herbarium; 1997. Department of Systematic Botany, Uppsala, Sweden.

29. Edwards S, Tadesse M, Demissew S, Hedberg I (Eds): Magnoliaceae to Flacourtiacea. Flora of Ethiopia and Eritrea Volume 2 Issue part 1. Addis Ababa, Ethiopia: The National Herbarium; 2000. Department of Systematic Botany, Uppsala, Sweden

30. Hedberg I, Edwards S (Eds): Pittosporaceae to Araliaceae. Flora of Ethiopia Volume 3. Addis Ababa, Ethiopia: The National Herbarium; 1989. Department of Systematic Botany, Uppsala, Sweden.

31. Hedberg I, Edwards S, Nemomissa S (Eds): Apiaceae to Dipsacaceae. Flora of Ethiopia and Eritrea Volume 4 Issue part 1. Addis Ababa, Ethiopia: The National Herbarium; 2003. Department of Systematic Botany, Uppsala, Sweden.

32. Hedberg I, Friis I, Edwards S (Eds): Asteraceae Volume 4. Issue part 2. Flora of Ethiopia and Eritrea. Addis Ababa, Ethiopia: The National Herbarium; 2004. Department of Systematic Botany, Uppsala, Sweden.

33. Hedberg I, Kelbesa E, Edwards S, Demissew S, Persson E (Eds): Gentianaceae to Cyclocheilaceae Volume 5. Flora of Ethiopia and Eritrea. Addis Ababa, Ethiopia: The National Herbarium; 2006. Department of Systematic Botany, Uppsala, Sweden.

34. The plant list a working list of all plant species. In; 2013. (accessed date) Www.Theplantlist.Org. A working list of all plant species, Accessed date 2013.

35. Höft M, Barik SK, Lykke AM: Quantitative Ethnobotany. Applications of multivariate and statistical analyses in ethnobotany. People and Plant Working Paper; 1999.

36. Tabuti JRS, Dhillion SS, Lye KA: The status of wild food plants in Bulamogi County, Uganda. Int J Food Sci Nutr 2004, 55(6):485-498.

37. Pallant J: SPSS survival manual, a step by step guide to data analysis using SPSS for Windows. 3rd edition; 2007

38. Ascariasis: 2013. (accessed date) http://www.stanford.edu/class/humbio103/ ParaSites2005/Ascaris/JLora_ParaSite.htm. Accessed date 2013.

39. WHO: Water Sanitation Health- Water related diseases. WHO/WSH/ WWD/ DFS.01; 2013.

40. Lulekal $\mathrm{E}$, Kelbessa $\mathrm{E}$, Bekele $\mathrm{T}$, Yineger $\mathrm{H}$ : An ethnobotanical study of medicinal plants in Mana Angetu District, south-eastern Ethiopia. J Ethnobiol Ethnomed 2008, 4:1-10.

41. Mesfin F, Demissew S, Teklehaymanot T: An ethnobotanical study of medicinal plants in Wonago Woreda, SNNPR Ethiopia. J Ethnobiol Ethnomed 2009, 5(28):1-18.
42. Togola A, Diallo D, Dembele S, Barsett H, Paulsen BS Ethnopharmacological survey of different uses of seven medicinal plants from Mali, (West Africa) in the regions Doila, Kolokani and Siby. J Ethnobiol Ethnomed 2005, 1(7):1-9.

43. Otieno NE, Analo C: Local indigenous knowledge about some medicinal plants in and around Kakamega forest in western Kenya. F1000Res 2012, 1(40):1-17.

44. Giday M, Teklehaymanot T: Ethnobotanical study of plants used in management of livestock health problems by Afar people of Ada'ar District, Afar Regional State Ethiopia. J Ethnobiol Ethnomed 2013, 9(8):1-10.

45. NCAPD: Seeking solutions for traditional herbal medicine: Kenya develops a national policy. Nairobi, Kenya: National Coordinating Agency for Population \& Development; 2008. Policy brief No.1.

46. Cunningham AB: African medicinal plants: Setting priorities at the interface between conservation and primary health care. People Plants Working Paper 1993, 1:1-50.

doi:10.1186/1746-4269-10-46

Cite this article as: Kidane et al.: Use and management of traditional medicinal plants by Maale and Ari ethnic communities in southern Ethiopia. Journal of Ethnobiology and Ethnomedicine 2014 10:46.

\section{Submit your next manuscript to BioMed Central and take full advantage of:}

- Convenient online submission

- Thorough peer review

- No space constraints or color figure charges

- Immediate publication on acceptance

- Inclusion in PubMed, CAS, Scopus and Google Scholar

- Research which is freely available for redistribution

Submit your manuscript at www.biomedcentral.com/submit
C BioMed Central 